


\title{
Moedas hispano-romanas do Gabinete de Numismática da Câmara Municipal do Porto
}

\author{
Autor(es): $\quad$ Pereira, Isabel
}

Publicado por: Imprensa da Universidade de Coimbra

URL persistente:

URI:http://hdl.handle.net/10316.2/45722

DOI:

DOI:https://dx.doi.org/10.14195/1647-8657_19_2

Accessed : $\quad$ 26-Apr-2023 12:30:49

A navegação consulta e descarregamento dos títulos inseridos nas Bibliotecas Digitais UC Digitalis, UC Pombalina e UC Impactum, pressupõem a aceitação plena e sem reservas dos Termos e Condições de Uso destas Bibliotecas Digitais, disponíveis em https://digitalis.uc.pt/pt-pt/termos.

Conforme exposto nos referidos Termos e Condições de Uso, o descarregamento de títulos de acesso restrito requer uma licença válida de autorização devendo o utilizador aceder ao(s) documento(s) a partir de um endereço de IP da instituição detentora da supramencionada licença.

Ao utilizador é apenas permitido o descarregamento para uso pessoal, pelo que o emprego do(s) título(s) descarregado(s) para outro fim, designadamente comercial, carece de autorização do respetivo autor ou editor da obra.

Na medida em que todas as obras da UC Digitalis se encontram protegidas pelo Código do Direito de Autor e Direitos Conexos e demais legislação aplicável, toda a cópia, parcial ou total, deste documento, nos casos em que é legalmente admitida, deverá conter ou fazer-se acompanhar por este aviso.

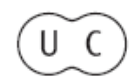


FACULDADE DE LETRAS

INSTITUTO DE ARQUEOLOGIA

CONIMBRIGA

VOLUME XIX

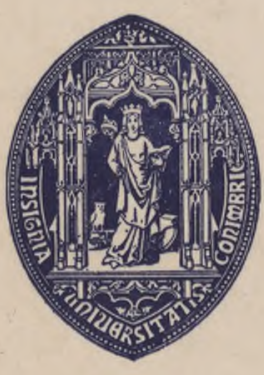

UNIVERSIDADE DE COIMBRA

1980 
ISABEL PEREIRA

Directora do Museu Municipal da Figueira da Foz

MOEDAS HISPANO-ROMANAS DO GABINETE DE NUMISMÁTICA DA CÂMARA MUNICIPAL DO PORTO

Conimbriga, XIX, 1980, 129-145

Resumo: As moedas hispano-romanas do Gabinete de Numismática da Câmara Municipal do Porto não têm indicação de proveniência. Não podemos assegurar que tenham resultado de achados feitos no norte de Portugal. A sua publicação parece, apesar de tudo, útil. São 189 peças, que nos quadros 1 e 2 se comparam com as moedas das mesmas épocas existentes nos museus de Antropologia do Porto, Monográfico de Conimbriga e Municipal da Figueira da Foz.

RÉSUMÉ: On ne connaît pas la provenance des monnaies hispano-romaines

du Cabinet des Monnaies de Câmara Municipal de Porto. Il est possible, mais pas sûr, qu'elles proviennent, pour la plupart, de trouvailles faites dans le nord du Portugal. Même avec cette incertitude, qui réduit son intérêt historique, la publication de ces monnaies paraît utile. C'est une collection de 189 pièces qui, dans les tableaux 1 et 2 sont comparées aux monnaies de même époque dans les musées de Conimbriga, Figueira da Foz et musée d'Anthropologie de la Faculté des Sciences de Porto. 
(Página deixada propositadamente em branco) 


\section{MOEDAS HISPANO-ROMANAS DO GABINETE DE NUMISMÁTICA DA CÂMARA MUNICIPAL DO PORTO}

\section{INTRODUÇÃO:}

Para o conhecimento da circulação monetária antiga, no nosso país, é urgente o estudo e publicação das colecções dos Museus, Gabinetes e demais instituições oficiais e particulares.

Com a publicação das moedas hispano-romanas existentes no Gabinete de Numismática da Câmara Municipal do Porto iniciaremos assim esse estudo.

Infelizmente, aqui, os registos são deficientes. Não mencionam a proveniência das peças, as condições de recolha e outro material arqueológico que porventura tenha sido encontrado associado aos numismas. Não fazem igualmente referência se as peças foram adquiridas, doadas ou recolhidas. Mencionam exclusivamente a denominação, o número de exemplares existentes, o peso e a referência a catálogos bibliográficos de fácil acesso ${ }^{\mathrm{x}}$ ). Mas só as espécies consideradas dignas de colecção tinham este tratamento. As outras eram registadas e agrupadas em lotes com a menção do peso total do lote $\left(^{2}\right)$. Deste modo, é-nos impossível separar conjuntos e tesouros de que possuímos indicações bibliográficas seguras $\left(^{3}\right)$.

$\left.\mathrm{f}^{1}\right)$ No nosso caso, a referência das peças é dada em relação à obra de Heiss (A), Description générale des monnaies antiques d'Espagne, Paris, 1870.

${ }^{2}$ As espécies de várias proveniências e consideradas «impróprias para colecção» eram agrupadas em grandes lotes, pesadas e registadas com a 
Pensamos todavia que, na generalidade, estas peças foram adquiridas em Portugal $\left(^{4}\right)$ e que até possivelmente foram encontradas em estações arqueológicas sitas no Norte do País. Dada a grande mobilidade do mercado numismático, outras hipóteses podem, contudo, ser admitidas.

O seu estado de conservação pode ser considerado bom, não levantando problemas de classificação.

Dado a pequena quantidade de exemplares examinados por centro de emissão - cidade - não realizaremos aqui estudos de metrologia, embora reconheçamos a sua necessidade para estas espécies em especial.

Nas conclusões finais, e como elemento de comparação, utilizaremos estudos já realizados e relativos às espécies de Conimbriga $\left({ }^{5}\right)$, Museu de Antropologia da Sociedade Mendes Correia, Porto $\left(^{6}\right)$ e às espécies ainda não publicadas do Museu Municipal «Dr. Santos Rocha», Figueira da Foz $\left({ }^{7}\right)$.

Neste último Museu, a constituição da colecção resulta no essencial de doações ( ${ }^{8}$ ). A maior e mais importante, no nosso caso, deve-se ao Dr. Mesquita de Figueiredo, longos anos residente em Lisboa e coleccionador afamado.

menção final do peso que oscilava, no Gabinete do Porto, sempre entre 3 a $5 \mathrm{Kg}$ e registadas no livro de Inventário com um só número. No caso das moedas romanas, muitos destes exemplares podem ser classificados até ao máximo pormenor.

$\left({ }^{3}\right)$ Castro hipólito (M. de), Dos tesouros de moedas romanas em Portugal, "Conimbriga», II-III, 1960-1961, p. 19 , n. ${ }^{\circ} 15$, p. 54, n. ${ }^{\circ} 70$, p. 59-60, n. ${ }^{\circ} 80$.

$\left.{ }^{4}\right)$ As moedas hispano-romanas, em bronze, foram exemplares pouco procurados por coleccionadores na época da formação do Gabinete. O contrário acontecia com as peças de prata.

(5) Pereira (J.), Bost (J. P.), hiernard (J.), Fouilles de Conimbriga, III. Les monnaies, Paris, 1974, p. 8-12.

(6) Centeno (R. M. S.), Moedas Hispano-Romanas no Museu de Antropologia «.Dr. Mendes Corrêa*», Porto, 1976, Trabalhos do Instituto de Antropologia «Dr. Mendes Corrêa», Faculdade de Ciências do Porto, n. ${ }^{\circ} 32$.

$\left(^{7}\right)$ O estudo da colecção do Museu «Dr. Santos Rocha», Figueira da Foz, iniciou-se cerca de 1971 pelo Dr. Mário de Castro Hipólito.

$\left.{ }^{8}\right)$ No que respeita às espécies numismáticas hispano-romanàs e romanas, os dois grandes doadores foram o fundador do Museu, Dr. Santos Rocha e o Dr. Mesquita de Figueiredo. 
Embora os registos do Museu sejam também omissos, pensamos que foram peças adquiridas em Lisboa e que não têm a mínima relação com as estações arqueológicas do centro do País, tão bem representadas no Museu da Figueira da Foz.

\section{Abreviaturas}

G. F. - Farres (O.G.), La Moneda Hispánica en la Edad Antigua, Madrid, 1966.

gr. - Gramas.

n. ${ }^{\circ} \quad$-Número de ordem das peças

V - Vives y Escudero (A), La Moneda Hispanica, 4 tomos e 1 volume de estampas, Madrid, 1924-1926. 


\section{CATÁlOGO}

EMISSÕES CARTAGINESAS NA HISPANIA

$A-$ Gadir (? $238-206$ ? A. C.)

\begin{tabular}{|c|c|c|c|}
\hline N. ${ }^{\circ}$ da moeda & Valor & Metal & \multicolumn{1}{c|}{ Observações } \\
\hline 1,2 & Calco & Cobre & 2 gr. Y $-10 / 23$ G. F. 67 \\
3 & $1 / 2$ Calco & Cobre & 1 gr. Y-10/18 G. F. 70 \\
4,5 & $1 / 4$ Calco & Cobre & 1 gr. V -9/10 G. F. 64 \\
6 & $1 / 4$ ou $1 / 8$ & & \\
& Calco & Cobre & 1 gr. V - 10/1 a 5 G. F. 57 \\
7 & $1 / 8$ Calco & Cobre & 1 gr. V-9/11 G. F. 65 \\
\hline
\end{tabular}

HISPANIA DURANTE A FASE UNCIAL

Hispania Citerior

$A$--Provavelmente Emporiae (? 120-90 A. C.)

\begin{tabular}{|l|l|l|l|}
\hline 8 a 10 & Is & Cobre & 16 gr. V-61/11 e 62/2 G. F. 272 \\
\hline
\end{tabular}

$B$ - Saguntum - Arse (? 190-90 A. C.)

\begin{tabular}{|c|c|c|c|}
\hline 11 & As & Cobre & 16 gr. V-18/6 G. F. 337 \\
12 & Quadrante & Cobre & 2 gr. V-19/6 G. F. 341 \\
13,14 & Quadrante & Cobre & 4 gr. V - 19/7 G. F. 344 \\
15 & Quadrante? & Cobre & 4 gr. V - ? G. F. ? \\
\hline
\end{tabular}

C - Valentia (? 120-90 A. C.)

\begin{tabular}{|l|l|l|l|}
\hline 16 & As & Cobre & 17 gr. V-125/1 G. F. 366 \\
\hline
\end{tabular}

Conimbriga, 19 (1980), 129-145 
Hispania Ulterior

$A$ - Castulo (? $120-90$ A. G.)

\begin{tabular}{|c|c|c|c|}
\hline N. ${ }^{\circ}$ da moeda & Valor & Metal & Observações \\
\hline 17,18 & Dupondio & Cobre & 27 gr. V-68/1 G. F. 378 \\
19 & Dupondio & Cobre & 19 gr. V-69/8 G. F. 383 \\
20 a 22 & As & Cobre & 13 gr. Y -69/2 G. F. 380 \\
23,24 & Semis & Cobre & 7 gr. V-68/12 G. F. 388 \\
25 & Semis & Cobre & 5 gr. Y -70/3 G. F. 390 \\
26,27 & Semis & Cobre & 8 gr. V -71/2 G. F. 395 \\
28 & Semis & Cobre & 9 gr. V-71/7 G. F. 398 \\
& & & \\
\hline
\end{tabular}

Obulco (? $120-90$ A. G.)

\begin{tabular}{|l|ll|l|}
\hline 29 a 31 & As & Cobre & 19 gr. V $-95 / 5$ G. F. 418 \\
\hline
\end{tabular}

A HISPANIA CITERIOR ENTRE $100-50$ A. G.

1 -EMISSÕES DE PRATA — JINETE IBÉRICO

$A$ - Incerta - (Ampurias ou Tarragona? 100-80 A. C.)

\begin{tabular}{|c|l|l|l|}
\hline 32 & Denário & Prata & 3 gr. V-40/11 G. F. 448 \\
33 & Denário & Prata & 4 gr. V-45/1 e 2 G. F. 454 \\
34 a 36 & Denário & Prata & 3 gr. V-43/2 G. F. 458 \\
37 & Denário & Prata & 3 gr. V-39/1 e 2 G. F. 464 \\
38 a 39 & Denário & Prata & 3 gr. V-66/2 G. F. 466 \\
40 & Denário & Prata & 4 gr. V-37/1 e 2 G. F. 474 \\
41 & Denário & Prata & 3 gr. V-51/7 G. F. 482 \\
\hline
\end{tabular}

2 - BRONZES LEGAIS DE 1.» SÉRIE - JINETE IBÉRICO

$A$ - Incertas - (Ampurias ou Tarragona - ? $100-80$ A. C.)

\begin{tabular}{|c|c|c|c|}
\hline 42 e 43 & As & Cobre & 10 gr. V-63/1 e 3 G. F. 515 \\
44 & As & Cobre & 11 gr. V-39/8 G. F. 612 \\
45 a 46 & As & Cobre & 13 gr. V-20/2 G. F. 668 \\
47 & As & Cobre & 9 gr. V-37/1 G. F. 688 \\
48 & Semis & Cobre & 4 gr. V-63/6 G. F. 518 \\
\hline
\end{tabular}

Conimbriga, 19 (1980), 129-145 
3 -EMISSÕES DEGENERADAS EM PRATA E BRONZE

Incerta $? 80-72$ A. G.

\begin{tabular}{|c|c|c|c|}
\hline N. ${ }^{\circ}$.da moeda & Valor & Metal & Observações \\
\hline 49 & Denário & Prata & 2 gr. V-47/1 e 3 G. F. 850 \\
\hline
\end{tabular}

A MOEDA DA HISPANIA CITERIOR $(50-23$ A. G. $)$

$A$ - Osca (39 - 27 A. C. - cunhagem de Cneo Domicio Calvino)

\begin{tabular}{|c|c|c|c|}
\hline 50 a 51 & Denário & Prata & 4 gr. V-136/1 F. F. 1002 \\
\hline \multicolumn{3}{|c|}{ B- Bilbilis (cunhagem local 28 A. C.) } \\
\hline 52 & As & Cobre & 12 gr. V-138/1 e 5 G. F. 1017 \\
\hline
\end{tabular}

$C-$ Calagurris (cunhagem local $33-25$ A. C.)

\begin{tabular}{|c|c|c|c|}
\hline 53 & As & Cobre & $33-28 ?$ A. C. \\
& As & Cobre & $\begin{array}{c}10 \text { gr. V }-157 / 7 \text { G. F. } 1028 \\
27-25 ? \text { A. C. } \\
11 \text { gr. V }-158 / 9 \text { G. F. } 1038\end{array}$ \\
\hline
\end{tabular}

D-- Carthagonova (cunhagem local $45-42$ ? A. C.)

\begin{tabular}{|c|l|l|l|}
\hline 55 a 56 & Semis & Cobre & 4 gr. V-130/1 G. F. 1046 \\
57 & Semis & Cobre & 3 gr. V-130/3 G. F. 1047 \\
58 & Semis & Cobre & 3 gr. V-130/2 G. F. 1049 \\
59 & Semis & Cobre & 8 gr. V-130/5 e 6 G. F. 1053 \\
\hline
\end{tabular}

Conimbriga, 19 (1980), 129-145 
$E-$ Celsa (cunhagem local $39-25$ ? A. C.)

\begin{tabular}{|c|c|c|c|}
\hline N. ${ }^{\circ}$ da moeda & Valor & Metal & Observações \\
\hline 60 & As & Cobre & $\begin{array}{c}39-37 \text { A. C. } \\
12 \text { gr. V }-160 / 1 \text { G. F. } 1057 \\
27-25 \text { A. C. } \\
61\end{array}$ \\
& As & Cobre & \begin{tabular}{c}
12 gr. V-161/2 G. F. 1069 \\
\hline
\end{tabular} \\
\hline
\end{tabular}

F- Ercavica (cunhagem local 27 A. C.)

\begin{tabular}{|c|c|c|l|}
\hline 62 & As & Cobre & 8 gr. y-162/3 G. F. 1105 \\
63 a 64 & Semis & Cobre & 5 gr. V-134/4 F. G. 1112 \\
65 & Semis & Cobre & 5 gr. V-133/2 G. F. 1119 \\
\hline
\end{tabular}

A MOEDA DA HISPANIA ULTERIOR $(100-23$ A. C. $)$

$A-$ Acinipo (cunhagem local $47-47$ A. C.)

\begin{tabular}{|l|l|l|l|}
\hline 66 & As & Cobre & 11 gr. V-105/11 e 15 G. F. 1162 \\
\hline
\end{tabular}

$B$ - Asido (cunhagem local $47-44$ A. C.)

\begin{tabular}{|l|l|l|l|}
\hline 67 & Semis & Cobre & 4 gr. V $-90 / 4$ G. F. 1170 \\
\hline
\end{tabular}

C-Carmo (cunhagem local 45 A. C.)

\begin{tabular}{|l|l|l|l|}
\hline 68 a 69 & As & Cobre & 7 gr. V-101/5 G. F. 1204 \\
\hline
\end{tabular}

$D$ - Carteia (cunhagem local desde 45 ? A. C.)

\begin{tabular}{|c|c|c|c|}
\hline 70 & Semis & Cobre & 11 gr. $V-120 / 12$ G. F. 1210 \\
\hline 71 a 73 & Semis & Cobre & $\begin{array}{l}4 \text { gr. V - } 126 / 8 \text { G. F. } 1229 \\
\text { Variante na legenda do reverso }\end{array}$ \\
\hline 74 & Semis & Cobre & 7 gr. V $-127 / 5$ G. F. 1238 \\
\hline 75 & Semis & Cobre & 4 gr. V $-127 / 6$ G. F. 1239 \\
\hline 76 & Semis & Cobre & 6 gr. V $-126 / 9$ G. F. 1242 \\
\hline
\end{tabular}

Conimbriga, 19 (1980), 129-145 
E- Castulo (cunhagem local 49 A. C.)

\begin{tabular}{|c|c|c|c|}
\hline N. ${ }^{\circ}$ da moeda & Valor & Metal & Observações \\
\hline 77 a 81 & As & Cobre & 12 gr. V $\_70 / 9$ G. F. 1247 \\
\hline
\end{tabular}

$F$ - Emerita (cunhagem local 23 A. C.)

\begin{tabular}{|c|c|c|c|}
\hline 82 a 83 & As & Cobre & 10 gr. V-140/14 G. F. 1289 \\
\hline
\end{tabular}

$G-$ Gades (cunhagem local $47-44$ A. C.)

\begin{tabular}{|c|c|c|l|}
\hline 84 a 89 & As & Cobre & 6 gr. V-74/1 G. F. 1297 \\
90 & Semis & Cobre & 4 gr. Inédita \\
91 a 94 & Semis & Cobre & 4 gr. V-74/5 G. F. 1300 \\
95 a 98 & Quadrante & Cobre & 1 gr. V $-74 / 7$ G. F. 1302 \\
99 & Quadrante & Cobre & 3 gr. V-74/10 e 12 G. F. 1305 \\
100 & Quadrante & Cobre & 2 gr. V-74/13? G. F. 1307? \\
\hline
\end{tabular}

$H$ - Ilipa (cunhagem local 47 - 44 A. C.)

\begin{tabular}{l|l|l|l|}
\hline 101 & Dupondio & Cobre & 19 gr. V -107/2 G. F. 1328 \\
\hline
\end{tabular}

1 - Incerta (situação desconhecida —• cunhagem local $47-44$ A. C.)

\begin{tabular}{|c|c|c|}
\hline 102 & As & Cobre 12 gr. V-71/14 G. F. 1366 \\
\hline
\end{tabular}

$J$ - Ituci (cunhagem local 47 - 44 A. C.)

\begin{tabular}{l|l|l|}
\hline 103 & As & Cobre 8 gr. V $-88 / 4$ G. F. 1387 \\
\hline
\end{tabular}

Conimbriga, 19 (1980), 129-145 
$L$ - Malaca - (cunhagem local 63 A. G.)

\begin{tabular}{|c|c|c|c|}
\hline$N^{\circ}$ da moeda & Valor & Metal & Observações \\
\hline 104 a 105 & As & Cobre & $\begin{array}{c}10 \text { gr. } \mathrm{y}-85 / 6,7 \mathrm{~V}-86 / 6,10, \\
15 \mathrm{G} . \mathrm{F} .1430 \\
6 \text { gr. Y }-85 / 9 \text { a } 14 \text { G. F. } 1441\end{array}$ \\
\hline
\end{tabular}

$M$ - Orippo (cunhagem local desde 38 A. G.)

\begin{tabular}{|c|c|c|c|}
\hline 108 & As & Cobre & 8 gr. V-110/1 G. F. 1493 \\
109 a 110 & As & Cobre & 5 gr. V-110/2 G. F. 1494 \\
111 & As & Cobre & 4 gr. V-110/3 G. F. 1496 \\
\hline
\end{tabular}

$N$ - Oset (cunhagem local 38 A. C.)

\begin{tabular}{|l|l|l|l|}
\hline 112 & As & Cobre & 5 gr. V-111/8 G. F. 1502 \\
\hline
\end{tabular}

EMISSÕES DO REINADO DE OCTÁVIO (23 A. C. 14 D. C.)

$A-\operatorname{Acci}(23$ A. C. -14 D. G.)

\begin{tabular}{|c|c|c|r|}
\hline $\begin{array}{c}113 \text { a } 114 \\
115\end{array}$ & As & Cobre & $\begin{array}{r}10 \text { gr. V }-166 / 5 \text { G. F. } 1581 \\
5 \text { gr. V }-166 / 6 \text { G. F. } 1582\end{array}$ \\
\hline
\end{tabular}

$B-$ Carteia (23 A. G. -14 D. G.)

\begin{tabular}{|c|l|l|l|}
\hline $\begin{array}{c}116 \text { a } 119 \\
120\end{array}$ & $\begin{array}{c}\text { Semis } \\
\text { Semis }\end{array}$ & $\begin{array}{c}\text { Cobre } \\
\text { Cobre }\end{array}$ & $\begin{array}{l}7 \text { gr. V-129/2 G. F. } 1625 \\
6 \text { gr. V-129/3, } 4 \text { G. F. } 1626\end{array}$ \\
\hline
\end{tabular}

Conimbriga, 19 (1980), 129-145 
C-Carthagonova (23 A. G. -14 D. G.)

\begin{tabular}{|c|c|c|c|}
\hline N. ${ }^{\circ}$ da moeda & Valor & Metal & Observações \\
\hline 121 & As & Cobre & $\begin{array}{c}12 \text { A. C. } \\
12 \text { gr. V-131/8 G. F. } 1636\end{array}$ \\
\hline 122 & As & Cobre & 1 gr. V-131/10 G. F. 1638 \\
\hline 123 a 124 & Semis & Cobre & $\begin{array}{c}\text { 19 A. C. } \\
4 \text { gr. V }-130 / 15 \text { G. F. } 1629\end{array}$ \\
\hline 125 a 126 & Semis & Cobre & $\begin{array}{l}23 \text { A. C. } \\
5 \text { gr. V }-131 / 9 \text { G. F. } 1637\end{array}$ \\
\hline 127 a 129 & Semis & Cobre & 6 gr. V $-131 / 12$ G. F. 1639 \\
\hline 130 a 131 & Semis & Cobre & 4 gr. V $-130 / 7$ G. F. 1644 \\
\hline
\end{tabular}

$$
E \text { - Gelsa (5 A. G.) }
$$

\begin{tabular}{|c|c|c|c|}
\hline 132 & As & Cobre & $V-161 / 8$ G. F. 1646 \\
\hline \multicolumn{4}{|c|}{$F$ - Ebora (12 A. C.) } \\
\hline 133 a 134 & As & Cobre & 6 gr. Y $-165 / 3$ G. F. 1650 \\
\hline \multicolumn{4}{|c|}{$G$ - Emerita (23 A. C. -14 D. C.) } \\
\hline $\begin{array}{l}135 \\
136\end{array}$ & $\begin{array}{l}\text { As } \\
\text { As }\end{array}$ & $\begin{array}{l}\text { Cobre } \\
\text { Cobre }\end{array}$ & $\begin{array}{l}10 \text { gr. } Y-141 / 6 \text { G. F. } 1660 \\
10 \text { gr. V }-141 / 4 \text { G. F. } 1674\end{array}$ \\
\hline \multicolumn{4}{|c|}{$H$ - Osca (1 A. C.) } \\
\hline 137 & As & Cobre & 11 gr. V-136/9 G. F. 1697 \\
\hline \multicolumn{4}{|c|}{ / - Patricia $(13-12$ A. C. $)$} \\
\hline 138 a 146 & As & Cobre & 9 gr. V $-165 / 3$ G. F. 1704 \\
\hline 147 a 148 & Semis & Cobre & 4 gr. V -165/6 G. F. 1705 \\
\hline
\end{tabular}

Conimbriga, 19 (1980), 129-145 


$$
J-\text { Traducta }-(13-12 \text { A. C. })
$$

\begin{tabular}{|c|c|c|c|}
\hline N. ${ }^{\circ}$ da moeda & Valor & Metal & Observações \\
\hline 149 a 155 & As & Cobre & 7 gr. V - 164/13 G. F. 1729 \\
\hline
\end{tabular}

MOEDAS DO REINADO DE TIBÉRIO (14 - 37 D. G.)

$A$-Calagurris $(14-37$ D. C. $)$

\begin{tabular}{|l|l|l|l|}
\hline 156 a 157 & As & Cobre & 9 gr. V-159/5 G. F. 1801 \\
\hline
\end{tabular}

$$
B \text { - Carteia (14 - } 37 \text { D. C.) }
$$

\begin{tabular}{|c|c|c|c|}
\hline 158 a 161 & Quadrante & Cobre & 19 D. C. \\
162 a 164 & Quadrante & Cobre & $\begin{array}{c}3 \text { gr. V }-128 / 14 \text { G. F. } 1805 \\
19 \text { D. C. }\end{array}$ \\
$\begin{array}{c}165 \\
166 \text { a } 168\end{array}$ & $\begin{array}{c}\text { Quadrante } \\
\text { Semis }\end{array}$ & $\begin{array}{c}\text { Cobre } \\
\text { Cobre }\end{array}$ & $\begin{array}{c}4 \text { gr. V }-128 / 11 \text { G. F. } 1806 \\
6 \text { gr. V }-128 / 2 \text { G. F. } 1809\end{array}$ \\
\hline
\end{tabular}

$$
\text { C-Carthagonova (14 - } 37 \text { D. C.) }
$$

\begin{tabular}{|c|c|c|c|}
\hline 169 & As & Cobre & $\begin{array}{c}23 \text { D. C. } \\
11 \text { gr. V }-132 / 1 \text { G. F. } 1828 \\
4 \text { gr. V }-131 / 15 \text { G. F. } 1825\end{array}$ \\
\hline
\end{tabular}

$$
D \text { - Caseatum }(14-37 \text { D. C. })
$$

\begin{tabular}{|c|c|c|c|}
\hline 172 & As & Cobre & 9 gr. V- 161/1 G. F. 1834 \\
\hline \multicolumn{3}{|c|}{$E-$ Clunia $(14-37$ D. C. $)$} \\
\hline 173 & As & Cobre & 8 gr. V- $163 / 4$ G. F. 1824 \\
\hline
\end{tabular}

Conimbriga, 19 (1980), 129-145 
F- Emerita (14 - 37 D. C.)

\begin{tabular}{|c|c|c|c|}
\hline N. $^{\circ}$ da moeda & Valor & Metal & Observações \\
\hline 174 & Dupondio & Cobre & 15 gr. G. F. 1857 \\
175 & As & Cobre & 16 gr. GIF 99, 100 G. F. 1865 \\
\hline
\end{tabular}

$$
G \text { - Gracurris (14 - } 37 \text { D. C. })
$$

\begin{tabular}{|l|l|l|l|}
\hline 176 & As & Cobre & 9 gr. V-163/1 G. F. 1921 \\
\hline
\end{tabular}

$$
H-\text { Ilici }(14-37 \text { D. G. })
$$

\begin{tabular}{|c|c|c|c|}
\hline 177 & As & Cobre & 19 D. C. \\
178 a 179 & As & Cobre & 8 gr. V $-144 / 13$ G. F. 1923 \\
180 & Semis & Cobre & 22 D. C. \\
& & & 11 gr. V $-133 / 10$ G. F. 1926 \\
& & & 19 D. C. \\
& &
\end{tabular}

$$
\text { / - Saguntum (14 - } 37 \text { D. C. })
$$

181 a 182 I As

Cobre 11 gr. Y - 124/3 G. F. 1953

$$
J \text { - Turiaso (14 - } 37 \text { D. G.) }
$$

\begin{tabular}{|c|c|r|}
\hline 183 As & Cobre & 9 gr. V-156/7 G. F. 1984 \\
184 As & Cobre & gr. V-156/11 G. F. 1987 \\
\hline
\end{tabular}

MOEDAS DO REINADO DE CALIGULA (37 - 41 D. G.)

$A$ - Garthagonova $(37-39)$

\begin{tabular}{|c|c|c|}
\hline 185 a 186 & As & Cobre 10 gr. V-132/7 e 10 G. F. 2018 \\
\hline \multicolumn{2}{|c|}{$B-$ Segobriga $(37-41$ D. C. $)$} \\
\hline $\begin{array}{c}\text { ! } 187 \text { a } 188 \\
189\end{array}$ & $\begin{array}{c}\text { As } \\
\text { Semis }\end{array}$ & $\begin{array}{l}\text { Cobre j } 10 \text { gr. V }-135 / 9 \text { G. F. } 2031 \\
\text { Cobre } ! 5 \text { gr. } 135 / 8 \text { G. F. } 2032 \\
1\end{array}$ \\
\hline
\end{tabular}




\section{CONCLUSÕES:}

Examinando o quadro 1 concluímos que no séc. Ill a.C. - período das emissões cartaginesas em Espanha - só o Gabinete Numismático do Porto possui exemplares, 7, todos de Gades, representando $3,7 \%$ do total da colecção. Nem o Museu de Antropologia «Mendes Correia» - também do Porto, nem o Museu de Conimbriga, nem o Museu «Dr. Santos Rocha» da Figueira da Foz, possuem exemplares deste período.

Do período Uncial, na Hispania Citerior, o Gabinete de Numismática do Porto possui 9 exemplares $(4,7 \%)$ e o Museu «Dr. Santos Rocha», Figueira da Foz, possui 7 exemplares $(2,8 \%)$. Nem Conimbriga nem o Museu de Aptropologia «Dr. Mendes Correia» possuem exemplares.

$\mathrm{Na}$ Hispania Ulterior, para o mesmo período, já todos os Museus estão regularmente representados:

$\begin{array}{ll}\text { Gabinete de Numismática } & -15 \text { exemplares }-7,8 \% \\ \text { Museu de Antropologia } & -2 \text { exemplares- }-3,0 \% \\ \text { Conimbriga } & -5 \text { exemplares }-5,4 \% \\ \text { Museu «Dr. Santos Rocha» } & -11 \text { exemplares }-4,5 \%\end{array}$

Entre 100 a 50 a.C., na Hispania Citerior, o Gabinete de Numismática do Porto possuí 18 exemplares (9,5\%), o Museu de Antropologia não tem exemplares, o Museu de Conimbriga possui 1 exemplar (1\%) e o Museu da Figueira da Foz tem 35 exemplares (14 4\%).

Para a Ulterior, no mesmo período, o Gabinete de Numismática do Porto possuí 47 exemplares $(24,8 \%)$, o Museu de Antropologia 8 exemplares (12,1\%), o de Conimbriga 8 exemplares $(8,7 \%)$ e o Museu «Dr. Santos Rocha» 53 exemplares $(21,9 \%)$.

$\mathrm{Na}$ Ulterior, entre 100-23 a.C., o Gabinete de Numismática do Porto está representado com 47 exemplares (24,8\%), o Museu 
de Antropologia com 8 exemplares (12,1\%), o Museu de Conimbriga também com 8 exemplares $(8,7 \%)$ e o Museu «Dr. Santos Rocha» com 53 exemplares $(21,9 \%)$.

De P. Carisius (27 - 25a.C.), cunhagem de campanha, o Gabinete de Numismática do Porto não possui exemplares, o Museu de Antropologia possui 1 exemplar (1,5\%), o de Conimbriga possui 11 exemplares (12\%) e o Museu Municipal «Dr. Santos Rocha» possui 7 exemplares $(2,8 \%)$.

Com Octávio (23 A.C. a 14 D.C.) as percentagens aumentam em todas as colecções:

Gabinete de Numismática -43 exemplares $-\quad(22,7 \%)$
Museu de Antropologia -22 exemplares $-(33,3 \%)$
Conimbriga
Museu «Dr. Santos Rocha» -49 exemplares $-(20,2 \%)$

Sob Tibério (14-37 D.C.), o panorama apresenta-se do seguinte modo:

$\begin{array}{ll}\text { Gabinete de Numismática } & -29 \text { exemplares - }(15,3 \%) \\ \text { Museu de Antropologia } & -20 \text { exemplares }-(30,3 \%) \\ \text { Conimbriga } & -38 \text { exemplares }-(41,7 \%) \\ \text { Museu «Dr. Santos Rocha» } & -54 \text { exemplares }-(22,3 \%)\end{array}$

Com Caligula (37-41 D.C.), nota-se o seguinte:

Gabinete de Numismática do Porto

Museu de Antropologia

Conimbriga

Museu «Dr. Santos Rocha»
- 5 exemplares - $(2,6 \%)$

- 1 exemplar - $(1,5 \%)$

- 1 exemplar - $(1,0 \%)$

- 7 exemplares - $(2,8 \%)$

Examinando as colecções por outro prisma verifica-se que os centros emissores (quadro 2) estão diferentemente representados nas colecções apresentadas. Só Emérita, na época de Octávio e Tibério, mantém lugar de destaque tanto em Conimbriga como na colecção do Museu «Dr. Santos Rocha» e relativamente impor- 
tante na colecção do Museu de Antropologia «Dr. Mendes Correia» do Porto.

A estrutura das quatro colecções aparece-nos tanto no quadro 1 como no quadro 2 com diferenças notáveis.

- Por períodos, os centros emissores estão diferentemente representados, em especial anteriormente ao reinado de Octávio.

- Por percentagem, dentro das grandes divisões periódicas tradicionaJmente aceites, é sensível as diferenças de colecção para colecção.

- Por centros emissores a representação é diferente em todas as colecções. Todavia, como já se disse, só Emérita, com Octávio e Tibério, está representada com certo equilíbrio.

Estas disparidades devem-se, naturalmente, aos diferentes processos de formação das colecções.

As moedas de Conimbriga, provenientes de uma estação arqueológica, científicamente escavada, reflectem de certo modo o panorama de circulação monetária local durante os períodos abrangidos. Tenha-se todavia em conta que o reduzido número de espécies e a extensão da escavação realizada não nos permitem fixar resultados definitivos.

Quanto às outras colecções, embora admitindo que comportam exemplares adquiridos em Portugal, o seu processo de formação foi lento e sujeito a conceitos numismáticos variáveis.

A compra, a troca, as doações, os legados e até os exemplares provenientes de escavações tudo se mistura em mostruários dignos de nota. Mas não traduzem, por isso, o panorama de circulação monetária da época. Mesmo assim, dão-nos contributos importantes e elementos de comparação indispensáveis quando, no futuro, todas as espécies provenientes de estações arqueológicas determinadas estiverem convenientemente estudadas e publicadas. 
(Página deixada propositadamente em branco) 
QUADRO 1

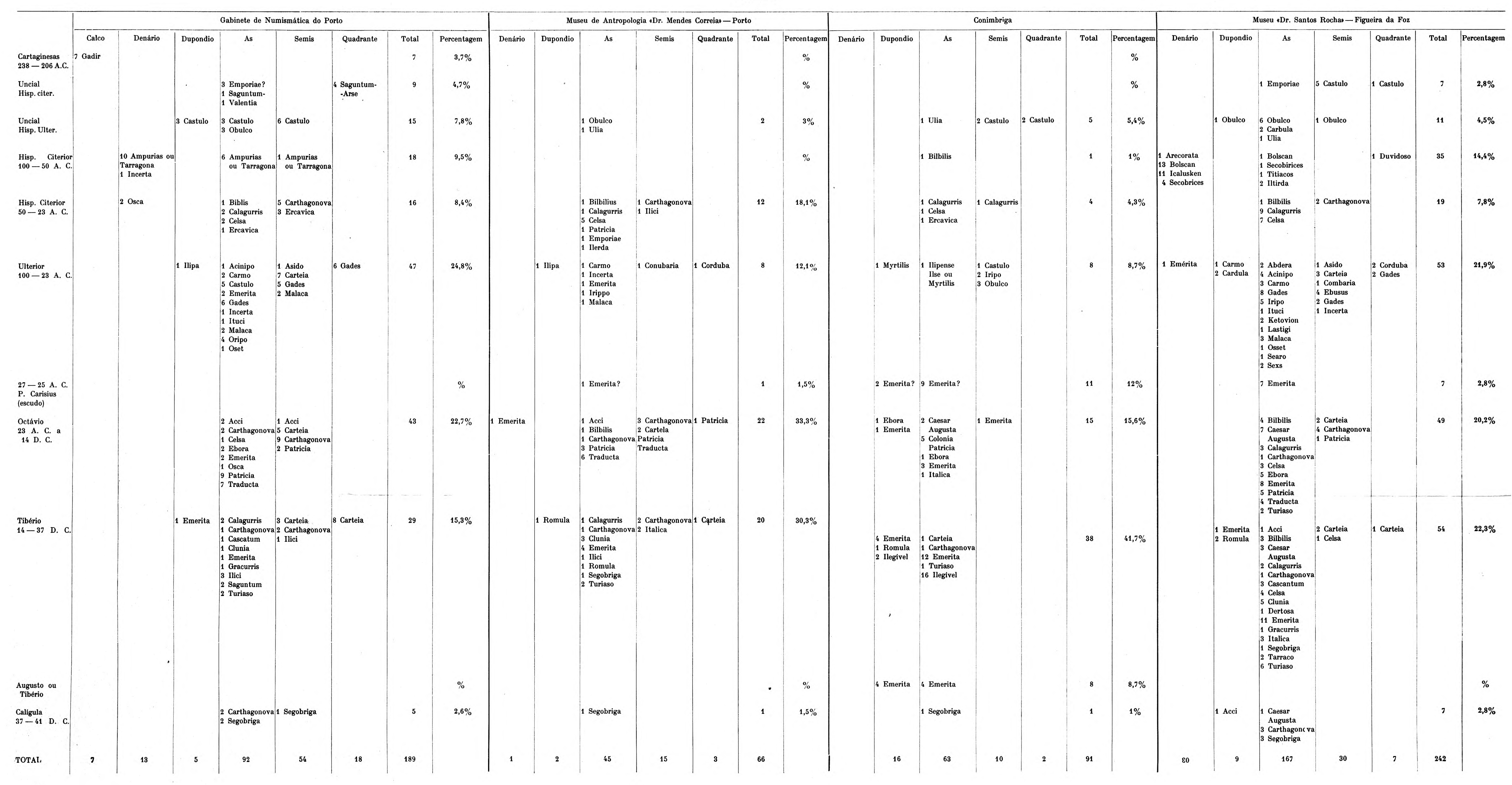


(Página deixada propositadamente em branco) 
QUADRO 2

GABINETE DE NUMISMÁTICA DO PORTO

\begin{tabular}{|c|c|c|c|c|c|c|c|c|c|c|}
\hline $\begin{array}{c}\text { Cartaginesas } \\
238-206 \text { A.C. }\end{array}$ & $\begin{array}{c}\text { Uncial } \\
\text { Hisp. Citerior }\end{array}$ & $\begin{array}{c}\text { Uncial } \\
\text { Hisp. Ulterior }\end{array}$ & $\begin{array}{l}\text { Hisp. citerior } \\
100-50\end{array}$ & $\begin{array}{l}\text { Hisp. citerior } \\
50-23\end{array}$ & $\begin{array}{l}\text { Ulterior } \\
100-23\end{array}$ & $\begin{array}{l}\text { P. Carisius } \\
27-25\end{array}$ & $\begin{array}{c}\text { Octávio } \\
23 \text { A.C. } 14 \text { D.C. }\end{array}$ & $\begin{array}{l}\text { Tibério } \\
14-37\end{array}$ & $\begin{array}{l}\text { Augusto } \\
\text { ou Tibério }\end{array}$ & $\begin{array}{l}\text { Calígula } \\
37-41\end{array}$ \\
\hline 7 Gadir & $\begin{array}{ll}4 & \text { Saguntum } \\
& \text { - Arse } \\
3 & \text { Emporiae } \\
1 & \text { Saguntum } \\
1 & \text { Valentia }\end{array}$ & $\begin{array}{l}12 \text { Castulo } \\
3 \text { Obulco }\end{array}$ & $\begin{array}{ll}17 \text { Ampurias ou } \\
\text { Tarragona } \\
1 \text { Incerta }\end{array}$ & \begin{tabular}{|ll}
5 & Carthagonova \\
4 & Ercavica \\
2 & Calagurris \\
2 & Celsa \\
2 & Osca \\
1 & Bilbilis
\end{tabular} & $\begin{aligned} 17 & \text { Gades } \\
7 & \text { Carteia } \\
5 & \text { Castulo } \\
4 & \text { Malaca } \\
4 & \text { Orippo } \\
2 & \text { Carmo } \\
2 & \text { Emerita } \\
1 & \text { Acinipo } \\
1 & \text { Asido } \\
1 & \text { Ilipa } \\
1 & \text { Ituci } \\
1 & \text { Incerta } \\
1 & \text { Oset }\end{aligned}$ & & $\begin{array}{l}11 \text { Carthagonova } \\
11 \text { Patricia } \\
7 \text { Traducta } \\
5 \text { Carteia } \\
3 \text { Acci } \\
2 \text { Ebora } \\
2 \text { Emerita } \\
1 \text { Celsa } \\
1 \text { Osca }\end{array}$ & $\begin{array}{ll}11 & \text { Carteia } \\
4 & \text { Ilici } \\
3 & \text { Carthagonova } \\
2 & \text { Calagurris } \\
2 & \text { Emerita } \\
2 & \text { Saguntum } \\
2 & \text { Turiaso } \\
1 & \text { Cascatum } \\
1 & \text { Clunia } \\
1 & \text { Gracurris }\end{array}$ & & $\begin{array}{ll}3 & \text { Segobriga } \\
2 & \text { Carthagonova }\end{array}$ \\
\hline
\end{tabular}

MUSEU DE ANTROPOLOGIA — «DR. MENDES CORREIA» - PORTO

\begin{tabular}{|c|c|c|c|c|c|c|c|c|c|}
\hline & $\begin{array}{ll}1 & \text { Obulco } \\
1 & \text { Ulia }\end{array}$ & & $\mid \begin{array}{ll}5 & \text { Celsa } \\
1 & \text { Bilbilis } \\
1 & \text { Calagurris } \\
1 & \text { Carthagonova } \\
1 & \text { Emporiae } \\
1 & \text { Ilirda } \\
1 & \text { Ilici } \\
1 & \text { Patricia }\end{array}$ & $\begin{array}{ll}1 & \text { Carmo } \\
1 & \text { Conubaria } \\
1 & \text { Corduba } \\
1 & \text { Emerita } \\
1 & \text { Ilipa } \\
1 & \text { Incerta } \\
1 & \text { Irippo } \\
1 & \text { Malaca }\end{array}$ & 1 Emérita? & $\begin{array}{ll}7 & \text { Traducta } \\
6 & \text { Patricia } \\
4 & \text { Carthagonova } \\
2 & \text { Carteia } \\
1 & \text { Acci } \\
1 & \text { Bilbilis } \\
1 & \text { Emérita }\end{array}$ & $\begin{array}{ll}4 & \text { Emérita } \\
3 & \text { Carthagonova } \\
3 & \text { Clunia } \\
2 & \text { Italica } \\
2 & \text { Romula } \\
2 & \text { Turiaso } \\
1 & \text { Calagurris } \\
1 & \text { Carteia } \\
1 & \text { Ilici } \\
1 & \text { Segobriga }\end{array}$ & & 1 Segobriga \\
\hline \multicolumn{10}{|c|}{ CONIMBRIGA } \\
\hline & $\begin{array}{ll}4 & \text { Castulo } \\
1 & \text { Ulia }\end{array}$ & 1 Bilbilis & $\begin{array}{ll}2 & \text { Calagurris } \\
1 & \text { Celsa } \\
1 & \text { Ercavica }\end{array}$ & $\mid \begin{array}{ll}3 & \text { Obulco } \\
2 & \text { Irippo } \\
1 & \text { Castulo } \\
1 & \text { Myrtilis } \\
1 & \text { Ilipense } \\
& \text { Ilse ou } \\
& \text { Myrtilis }\end{array}$ & 11 Emerita? & $\left|\begin{array}{ll}5 & \text { Colónia Patr. } \\
5 & \text { Emérita } \\
2 & \text { Ebora } \\
2 & \text { Caesar } \\
& \text { Augusta } \\
1 & \text { Italica }\end{array}\right|$ & \begin{tabular}{|rl}
16 & Emerita \\
18 & Ilegíveis \\
1 & Carteia \\
1 & Carthagon. \\
1 & Turiaso \\
1 & Romula
\end{tabular} & 8 Emerita & 1 Segobriga \\
\hline \multicolumn{10}{|c|}{ MUSEU MUNICIPAL «DR. SANTOS ROCHA»-FIGUEIRA DA FOZ } \\
\hline $\begin{array}{ll}6 & \text { Castulo } \\
1 & \text { Emporiae }\end{array}$ & $\mid \begin{array}{ll}8 & \text { Obulco } \\
2 & \text { Carbula } \\
1 & \text { Ulia }\end{array}$ & \begin{tabular}{|rl}
14 & Bolscon \\
11 & Icalgunkum \\
5 & Secobirices \\
2 & Iltirda \\
1 & Arecorata \\
1 & Titiacos \\
1 & Duvidoso
\end{tabular} & $\mid \begin{array}{ll}9 & \text { Calagurris } \\
7 & \text { Celsa } \\
2 & \text { Carthagonova } \\
1 & \text { Bilbilius }\end{array}$ & \begin{tabular}{|rl}
12 & Gades \\
5 & Irippo \\
4 & Acinipo \\
4 & Carmo \\
4 & Ebusus \\
3 & Carteia \\
3 & Malaca \\
2 & Abdera \\
2 & Cordula \\
2 & Corduba \\
2 & Ketovion \\
2 & Sexs \\
1 & Asido \\
1 & Emerita \\
1 & Incerta \\
1 & Ituci \\
1 & Lastigi \\
1 & Conbaria \\
1 & Osset \\
1 & Searo
\end{tabular} & 7 Emerita & \begin{tabular}{|ll}
8 & Emerita \\
7 & Caesar \\
& Augusta \\
6 & Patricia \\
5 & Carthagonova \\
5 & Ebora \\
4 & Bilbilis \\
4 & Traducta \\
3 & Calagurris \\
3 & Celsa \\
2 & Carteia \\
2 & Turiaso
\end{tabular} & $\begin{aligned} 12 & \text { Emerita } \\
6 & \text { Turiaso } \\
5 & \text { Celsa } \\
5 & \text { Clunia } \\
3 & \text { Carteia } \\
3 & \text { Bilbilis } \\
3 & \text { Cascatum } \\
3 & \text { Caesar } \\
& \text { Augusta } \\
3 & \text { Italica } \\
2 & \text { Calagurris } \\
2 & \text { Romula } \\
2 & \text { Tarraco } \\
1 & \text { Acci } \\
1 & \text { Carthagon. } \\
1 & \text { Dertosa } \\
1 & \text { Gracurris } \\
1 & \text { Segobriga }\end{aligned}$ & . & $\begin{array}{ll}3 & \text { Carthagonova } \\
2 & \text { Segobriga } \\
1 & \text { Acci } \\
1 & \text { Caesar } \\
& \text { Augusta }\end{array}$ \\
\hline
\end{tabular}

